Document downloaded from:

http://hdl.handle.net/10251/136406

This paper must be cited as:

Climente Alarcon, V.; Arkkio, A.; Antonino-Daviu, J. (2018). 2-D Magnetomechanical Transient Study of a Motor Suffering a Bar Breakage. IEEE Transactions on Industry Applications. 54(3):2097-2104. https://doi.org/10.1109/TIA.2018.2791918

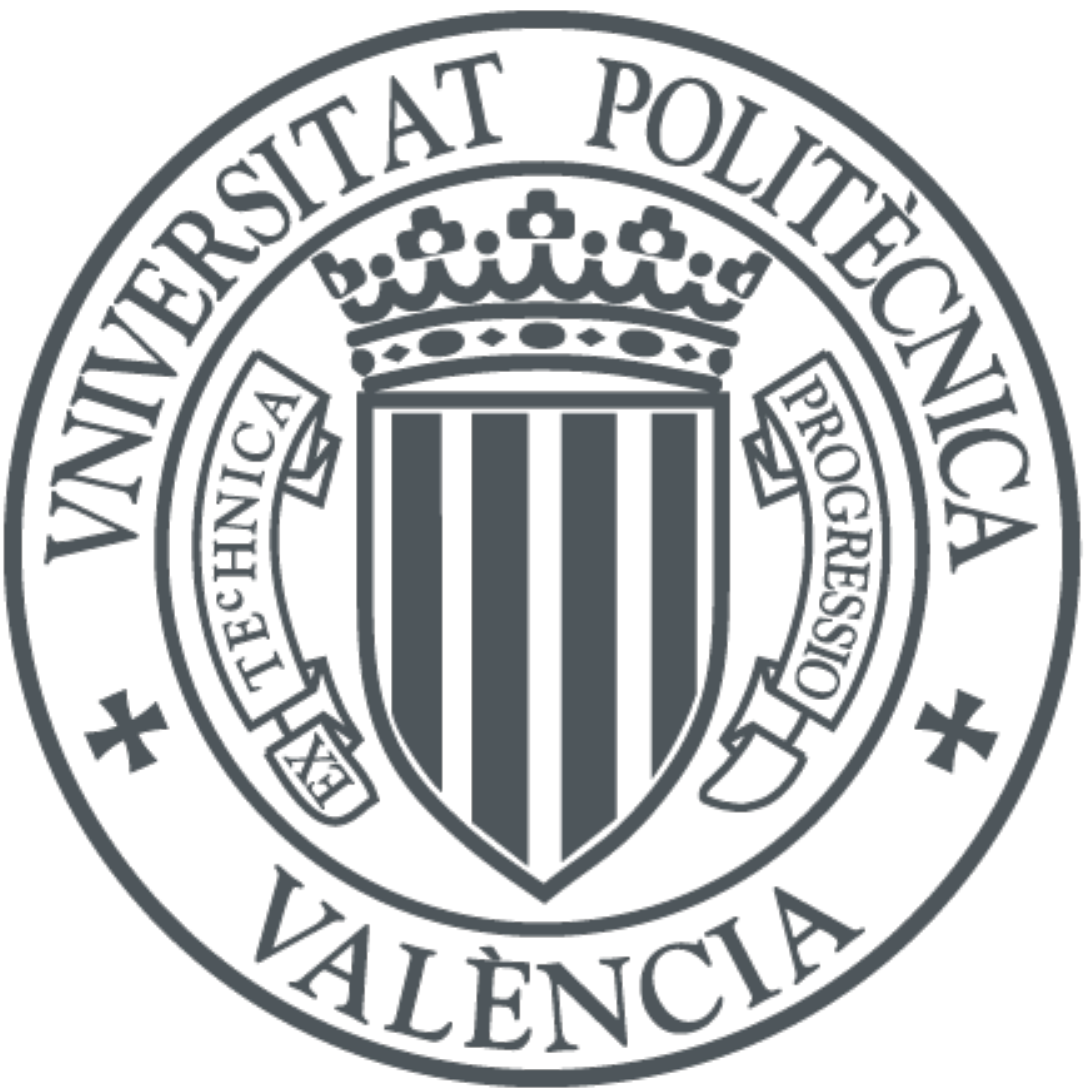

The final publication is available at

https://doi.org/10.1109/TIA.2018.2791918

Copyright Institute of Electrical and Electronics Engineers

Additional Information 


\section{2-D Magnetomechanical Transient Study of a Motor Suffering a Bar Breakage}

\author{
Vicente Climente-Alarcon, Antero Arkkio \\ Dept. of Electrical Engineering and Automation \\ Aalto University \\ Espoo, FINLAND \\ viclial@ieee.org, antero.arkkio@aalto.fi
}

\author{
José Antonino-Daviu \\ Instituto Tecnológico de la Energía, ITE \\ Universitat Politècnica de València \\ Valencia, SPAIN \\ joanda@die.upv.es
}

\begin{abstract}
The analysis of the vibration response of electrical machines has importance in noise prediction and more recently, diagnosis of electrical faults, especially in the industrial environment, where it is a well-known technique. This work assesses the performance of a strongly coupled 2-D magnetomechanical approach, as directly available in multiphysics software, for the simulation of an induction machine under heavy operational conditions: a direct-on-line startup. Both healthy and broken bar states are simulated in a time span long enough to allow the detailed study of the varying frequency components. The results yield, in addition to the usual electrical and magnetic quantities, electromagnetic-induced vibration components in the stator. A comparison with current and vibration experimental data is also performed showing a good agreement with variable frequency components and certain limitations concerning their amplitude.
\end{abstract}

Keywords-induction motors, finite element analysis, fault diagnosis, rotors, vibrations, multiphysics.

\section{INTRODUCTION}

The diagnosis of rotating equipment in general and more specifically rotating electrical machines by examining its vibrational response is a well establish technique advantageously applied in the industry for its non-invasive nature, effectiveness in detecting and tracking defects, such as bearing faults [1, 2] and shorted rotor laminations [3], and conceptual simplicity, since it is related to the sound emission of such equipment.

Nevertheless, the study of the effect of electrical faults in the vibration characteristics of the machine is troublesome, due to its complex construction, with laminated cores, slots and either wound or encased conductors, the interaction between mechanical and electromagnetic phenomena, and the influence of temperature changes in the response of the system [4].

Comprehensibly, seminal works on fault diagnosis of induction motors used the analytical study of the airgap flux permeance components to assess the impact of eccentricity in the machine's time and spatial vibration waves [5]. In this case the influence of the system's mechanical response to the magnetic force produced by those additional harmonics is not taken into account, just assuming that those same components exciting the structure from the airgap will appear on the external frame of the motor. Despite of this, several predicted frequencies and spatial waves could be detected in the experimental validation. A similar approach was followed in [6] where, due to the increasing availability of computational resources, a 2-D finite element simulation was performed instead to compute the electromagnetic state of an induction motor suffering electrical faults, the stress waves created by the radial magnetic flux density on the stator teeth were decomposed as a double Fourier series and the results experimentally validated.

A full consideration of the stator structure, able to predict not only the frequencies of some harmonics but the complete response of the machine under stationary operation, was recently proposed. In [7] a weak coupling between the 2-D electromagnetic finite element computation and a detailed 3-D structural model of the stator and end shields, validated by modal analysis, was introduced. A projection algorithm linked both models. The results were able to reproduce saturation and loading effects on the vibration waveforms measured on the stator frame and predict its main spatial waves. These models were applied in [8] to the difficult task of assessing the amplitude variation of specific vibration components according to the severity of the fault, in this case a bar breakage. A good agreement was obtained when a small number of broken bars were studied. However, only frequencies between $87 \mathrm{~Hz}$ and $220 \mathrm{~Hz}$ in stationary operation were examined and the influence of the skewing of the bars was not considered.

Nevertheless, the current trend in the development of numerical formulations and computational procedures to predict the vibrations of an electrical machine aims at avoiding the excitation of the mechanical model on its inner surfaces using the Maxwell stress tensor (MST). Magnetic forces caused by the flux in the iron are computed instead and, in some cases, even the effect of magnetostriction is taken into account [9]. This later phenomenon macroscopically reflects the rearrangement of magnetic domains inside the material when the direction of the magnetic field is varied and its comprisal would yield more accurate results concerning the amplitude of the vibrations generated. Despite all these recent improvements and with the aim of performing a long time-stepping simulation able to reproduce a heavy transient in an induction machine, in this work it was preferred to maintain the relative simplicity of a 2D magnetomechanical finite element analysis directly available in multiphysics software, which reduces the computational cost and also has the advantage that can be strongly coupled to the 2D electromagnetic simulation by means of the MST.

During transients such as a direct-on-line startup, the motor is subjected to high mechanical and electrical stresses and incipient faults may be easier to detect [10], yet the analysis of quantities becomes more complicated as the frequency decomposition using the Fast Fourier Transform (FFT) is 
generally unsuitable to be applied to study rotor-generated components. Thus, several signal analysis techniques have been devised, tested and validated for diagnosis of motors under transient operation based on different time-frequency decomposition (TFD) tools, such as discrete [11] and continuous [12] Wavelets, the Hilbert Huang transform (HH) [13] and the Wigner-Ville (WVD) and Zhao-Atlas-Marks distributions (ZAM) [14-16], among others. Nonetheless, their application to the study of the complex vibrational response of a motor under such demanding conditions has been limited. In [17] the axial vibration of a double cage motor under Field Oriented Control (FOC) was examined with the purpose of diagnosing a bar breakage using an approach based on the discrete Wavelet transform. The ZAM is utilized in [16] to tackle the problem of analyzing a long startup in which sidebands around the Principal Slot Harmonics (PSH) were detected.

Thus, the aim of this work is to simulate the motor tested in [16] by means of a strongly coupled magnetomechanical 2-D model to reproduce the effects of a bar breakage during a directon-line (DOL) startup in electromagnetic and mechanical magnitudes. Both the frequency evolution and the amplitude of some of the components obtained are studied and compared to the experimental ones with the purpose of assessing the effectiveness of this approach and to have an insight into the electrical contribution to specific vibrational harmonics.

For this purpose, the remaining of this paper is structured as follows: Section II introduces in detail the computational method used to carry out the simulations, Section III explains the expected evolutions of the harmonics introduced by the bar breakage in the current and vibration spectra and summarizes the signal processing method employed to study them. Section IV presents the results and Section V yields the conclusions reached.

\section{COMPUTATIONAL PROCEDURE}

COMSOL commercial finite element analysis software has been used to perform two multiphysics simulations (healthy and one broken bar states) using two coupled solvers: rotating machinery (magnetic) and solid mechanics (linear elastic).

\section{A. Electromagnetic Analysis}

The conventional 2-D formulation for finite element analysis of electrical machines has been employed to obtain the electromagnetic state of the machine during the transient. Eq. 1, in which $\mu$ stands for the magnetic permeability of the corresponding material, $\sigma$ for its conductivity and $A$ for the component of the magnetic vector potential perpendicular to the computing plane, is solved along the circuit equations. The parameter $k$ takes a value of 0 in most of the mesh, except for the stator and rotor slots, where it equals the current density in the first case and a value dependent on the voltage difference at the bar ends for the second.

$$
-\nabla \cdot\left(\frac{1}{\mu} \nabla A\right)+\sigma \frac{\partial A}{\partial t}=k
$$

The electromagnetic torque is computed integrating the Maxwell stress tensor around the perimeter of the rotor and used in the movement equations to obtain its position in the next time step

$$
\boldsymbol{\tau}_{M S T}=\left(\begin{array}{cc}
B_{r}^{2}-\frac{1}{2}|\boldsymbol{B}|^{2} & B_{r} B_{t} \\
B_{r} B_{t} & B_{t}^{2}-\frac{1}{2}|\boldsymbol{B}|^{2}
\end{array}\right)
$$

where $B_{r}$ is the radial and $B_{t}$ the tangential component of the magnetic flux density.

\section{B. $\quad$ Solid mechanics analysis}

With the purpose of studying the effect of the bar breakage in the vibrational spectrum of the machine, a linear elastic finite element computation is applied to the stator iron. The equation to be solved is

$$
\rho \frac{\partial^{2} \boldsymbol{d}}{\partial t^{2}}-\nabla \cdot \boldsymbol{\tau}=\boldsymbol{f}
$$

where $\rho$ is the density, $\boldsymbol{d}$ the displacement field, $\boldsymbol{f}$ the given volume force and $\tau$ the stress tensor, which is related to the displacements by the strain $\boldsymbol{\varepsilon}$ and the elastic modulus $\boldsymbol{C}$, in this computation reduced to a scalar, since no anisotropy in the material was considered.

$$
\begin{gathered}
\boldsymbol{\tau}=\boldsymbol{C}: \boldsymbol{\varepsilon} \\
\boldsymbol{\varepsilon}=\frac{1}{2}\left(\nabla \boldsymbol{d}+(\nabla \boldsymbol{d})^{T}\right)
\end{gathered}
$$

The coupling between both solvers, electromagnetic and linear elastic, is carried out along the internal surfaces of the stator, setting as force boundary condition the output of the integration of the Maxwell stress tensor (2) on those same surfaces. In this particular case, the expression is simplified to just:

$$
\tau=\tau_{M S T}
$$

For the linear elastic analysis, silicon steel, with an elastic modulus of $193 \mathrm{GPa}$ and a Poisson's coefficient of 0.29 , was selected as the stator's material.

The setting of the corresponding values for the external surface in this 2-D simulation is not trivial, since the body of the motor is actually supported only at two small segments of its entire length. However, the movement of the rotor must be restrained in some way in order to find a solution to the linear elastic problem, and any realistic fastening condition can't be easily reproduced. Unlike [18], in this work the radial component of the displacement on the outer surface of the stator has been left free. Two probes were placed on the tip of a stator tooth and on its outer perimeter measuring in both radial and tangential direction displacements and, also for the second position, stresses (Fig. 1).

More than 3.3 seconds of the startup transient of a healthy motor and a motor having a broken bar have been simulated. The breakage was reproduced adding in the circuit equations of the rotor a series resistance on one bar with a value several orders of magnitude higher than the calculated bar resistance. The simulation took approximately one month in a normal PC. 


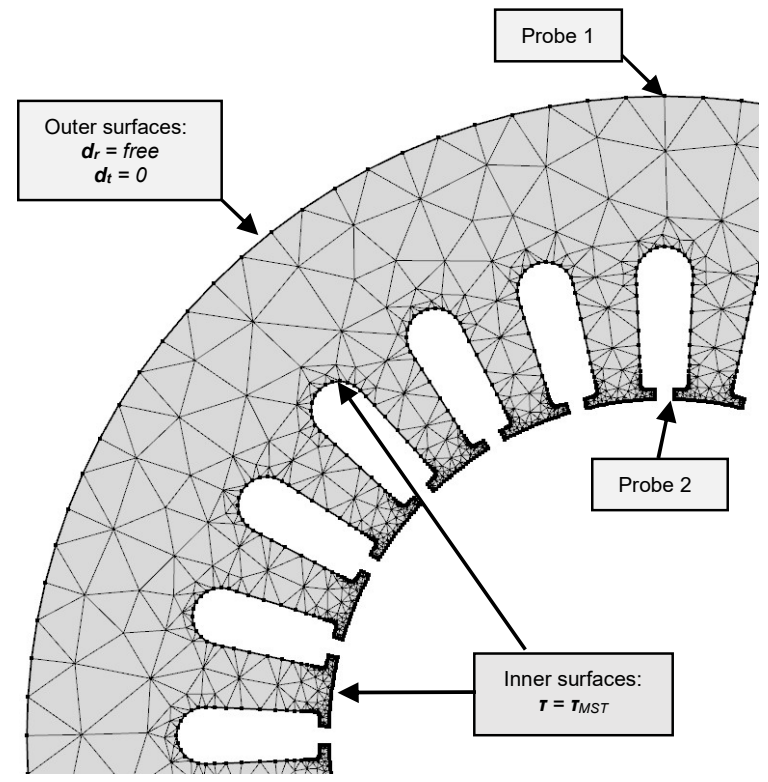

Fig. 1. Mesh, boundary conditions and location of the probes for the solid mechanics analysis of the stator.

\section{ANALYSIS PROCEDURE}

In this section the components introduced by the bar breakage in both the stator's vibration response and the stator's currents are presented, as well as a summary of the signal analysis procedure used to depict and quantify their variable frequency evolution during the DOL startup transient.

\section{A. Components arisen due to the asymmetry}

In [16] it was obtained, following the rotating field approach, an expression for the vibrational high frequency components evolution with respect to the slip $s$ :

$$
f_{v_{-} h i g h}(s)=\left[\left(k_{1} R\right) \frac{1-s}{p} \pm k_{2} 2 s \pm k_{3}\right] f
$$

where $R$ is the number of rotor bars, $p$ the number of pole pairs and $f$ is the stator's feeding frequency. This equation (7) depicts the rotor bar passing frequency (RBPF) and its harmonics $\left(k_{l}\right.$, $k_{3}$ ), for a healthy machine if $k_{2}=0$ (shown in Fig. 2 as (a1)). However, in the case a bar breakage exists, it was proved in [16] that the negative sequence component that arises due to this disturbance in the rotor m.m.f. produces additional sidebands with a characteristic evolution during a direct-on-line startup $\left(k_{2}\right.$ $\neq 0)(\mathrm{a} 2)$.

Furthermore, if there is no current flowing in one bar, its corresponding field doesn't exist and hence an unbalanced magnetic pull appears [4]. This induces further lower frequency vibration components with a frequency equal to the rotational frequency of the rotor and its harmonics (a3).

$$
f_{v_{-} \text {low }}(s)=k_{4} \cdot \frac{1-s}{p} f=k_{4} \cdot f_{r}
$$

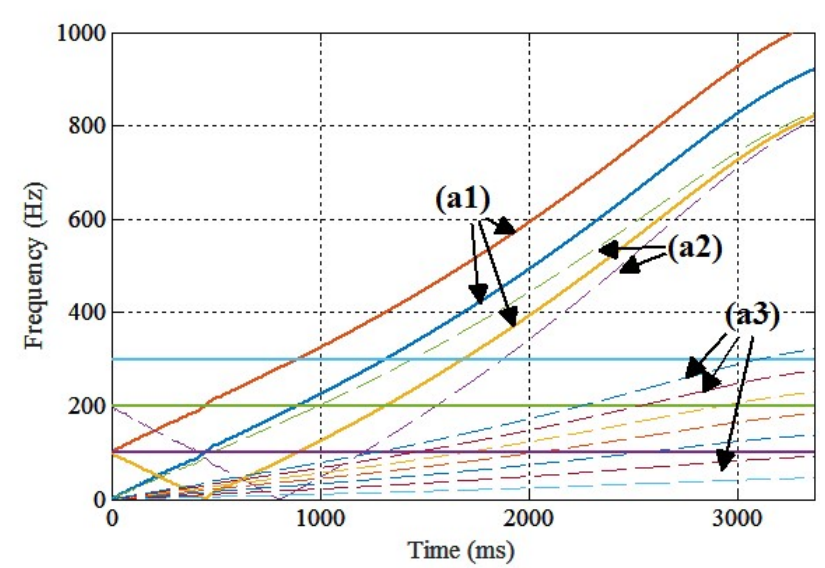

Fig. 2. Evolution of the variable frequency harmonics in the vibration spectrum of an induction motor during a DOL startup. Solid line for a healthy motor and dashed, additional components in case of a bar breakage. Constant frequency twice line component and its harmonics are also shown.

In the case of the stator currents, a general expression for the frequency of the components that the rotor asymmetry produces, also as a function of the $\operatorname{sip} s$, is [19]:

$$
f_{c_{-} 1}(s)=\left(\frac{k_{5}}{p}(1-s) \pm s\right) \cdot f \quad \frac{k_{5}}{p}=1,3,5 \ldots .
$$

From this equation, for each current harmonic of $k_{5} / p$ order, two components are obtained whose frequencies at steady state $(s \approx 0)$ are slightly smaller (b2, b4 in Fig. 3); one of such low sidebands (LSH) evolves directly ("+" sign in (9)) and the other evolves indirectly, first reducing its frequency and then increasing it again (“-” sign in (9)).

In addition, the torque pulsation caused by these low sidebands modulates the speed of the machine triggering further descending harmonics [20]. Therefore, for the main current component a full set of sidebands surrounding it would appear in the current spectrum during stationary operation (b3):

$$
f_{c_{-} 2}(s)=\left(1 \pm 2 \cdot k_{6} \cdot s\right) \cdot f \quad k_{6}=1,2,3 \ldots .
$$

Finally, in order to conclude the account of variable frequency harmonics, the Principal Slot Harmonics (PSH), related to the bar passing frequency and that under certain conditions also appear in the current spectrum, can be affected by the breakage (b1) [21]:

$$
f_{P S H \pm k 8}(\mathrm{~s})=\left[k_{7} \cdot R \frac{(1-s)}{\mathrm{p}} \pm k_{8}\right] \cdot f
$$

All these current components are summarized in Fig. 3.

All these vibration components are depicted in Fig. 2. 


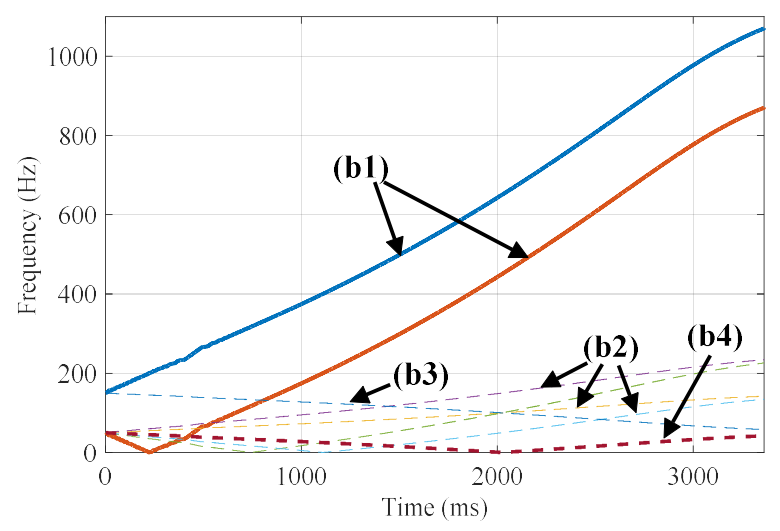

Fig. 3. Evolution of the variable frequency harmonics in the current spectrum of an induction motor during a DOL startup. Solid line for a healthy motor and dashed, additional components in case of a bar breakage. Winding harmonics are not depicted.

\section{B. Time-frequency analysis procedure}

As in [16] the vibration waveforms are analyzed using the Zhao-Atlas Marks distribution (ZAM) to avoid the effect of the cross terms or artifacts that appear between the components of the signal. This same tool has been employed to decompose the currents during the transient, in order to facilitate the comparison of results between both magnitudes.

$$
\begin{gathered}
Z A M_{X}(t, \omega)=\frac{1}{4 \pi a} \int_{-\infty}^{+\infty} g(\tau) e^{-j \tau \omega} \\
\int_{t-a|\tau|}^{t+a|\tau|} x\left(t+\frac{\tau}{2}\right) \cdot x^{*}\left(t-\frac{\tau}{2}\right) d t d \tau
\end{gathered}
$$

In (12) $x(t)$ is the signal studied, and $\tau$ is the delay variable. $g(\tau)$ and $a$ are a given function and given parameter that perform the filtering of the cross terms in the ambiguity plane.

Since the ZAM does not preserve the energy content of the signal, for the integration of the variable frequency harmonics' energy (an indication of the fault's severity) a procedure presented in [21] and based on the Wigner-Ville distribution (WVD) and particle filtering is preferred instead:

$$
W V D_{X}(t, \omega)=\frac{1}{2 \pi} \int_{-\infty}^{+\infty} x\left(t+\frac{\tau}{2}\right) \cdot x^{*}\left(t-\frac{\tau}{2}\right) e^{-j \tau \omega} d \tau .
$$

For the WVD the kernel is equal to one and therefore there is no filtering of crossterms by the distribution itself. These are tackled using Finite Impulse Response filters prior to the computation of (13). Advanced notch filters eliminate the constant frequency components, such as the main current one, and bandpass filters limit a time-frequency box where the evolution of one harmonic prevails. A Particle Filtering-based procedure tracks the evolution of the harmonic and accurately integrates the energy on its path. The comparison of this energy $e_{i f, b w}$ with the one in the original waveform $x(t)$ (Fig. 4-5) provides a numerical indicator of the fault:

$$
\gamma_{W, x}=10 \cdot \log \left[\frac{2 \cdot \sum_{t} x(t)^{2}}{e_{i f, b w}}\right]
$$

\section{RESULTS}

This section compares the output of the two simulations, corresponding to the healthy and broken bar states and also the later with the experimental results (in the case of vibrations, available from [16]). It is organized in five parts, beginning with a general assessment of the waveforms shape and amplitude, followed by their time-frequency analysis, a quantification of the energy present in some components used to diagnose the fault and finally a discussion of the results obtained.

\section{A. Time Domain Analysis}

Fig. 4 shows the waveforms computed in the simulation of the broken bar state for acceleration at Probe 1 (top, external perimeter of the stator) and Probe 2 (middle, tooth tip), and the stator current (bottom). Despite the modification of the boundary conditions, compared to [18], negligible radial displacements on the outer surface of the stator are detected. The waveform of Fig.4 (top) has been thus obtained calculating the displacement in the radial direction (for this point, vertical) according to the stress in that direction and the Young modulus. The result has been differentiated twice. Nevertheless, the radial acceleration at the Probe 2 location, the airgap, (Fig. 4, middle) corresponds to actual displacements obtained during the simulation.
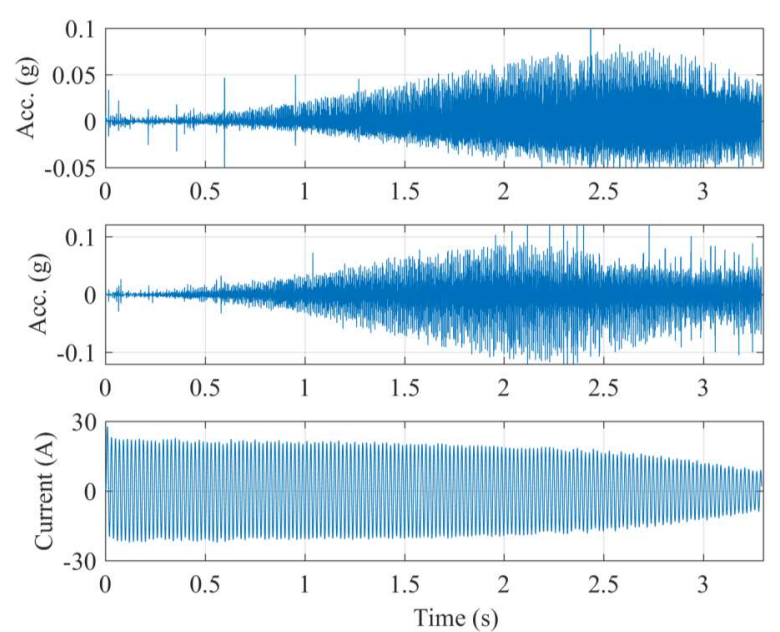

Fig. 4. Calculated acceleration waveform at the position of probe 1 (top), simulated acceleration at probe 2 (middle) and simulated stator current waveform (bottom) during the startup transient for the faulty state.

Fig. 5 shows the experimental data acquired in [16] from the same motor having a broken bar. The waveforms are longer because of the higher load inertia and also due to the fact that an extended part of the transient is shown. Yet, the overall shape of the vibration waveform (Fig. 5, top) is similar to the ones simulated (Fig. 4, top, middle), as the RBPF harmonics (7) reach higher frequencies and thus are amplificated by the better response of the accelerometer in this band. In addition, the contribution of mechanical-caused, rotational speed-related components (such as eccentricity or misalignment between motor and load), is shown at the beginning of the transient for the experimental signal as a higher amplitude value, whereas 
they are not depicted by the simulated ones, that only study electrical effects. Up to five resonant frequencies of the motor or the supports can be identified. They cannot be simulated either following this straightforward approach. Leaving aside these direct mechanical contributions and resonances, the overall amplitude of the simulated waveforms is approximately $25 \%$ of the actual values recorded during the experiment.
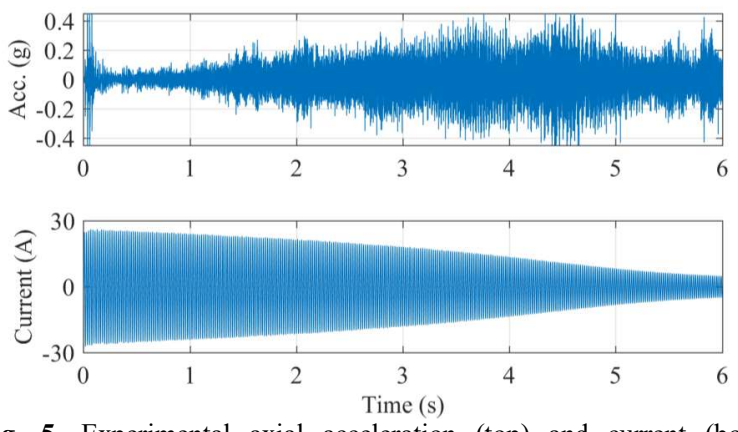

Fig. 5. Experimental axial acceleration (top) and current (bottom) waveforms recorded during the startup transient for the faulty state.

\section{B. Time-frequency analysis of the simulated results}

The analysis using the Zhao-Atlas-Marks distribution of the stress waves at the location of Probe 1 (Fig. 4, top) and stator current (Fig. 4, bottom) waveforms for both cases, healthy and broken bar, is presented in Fig. 6. Healthy states are displayed on the left-hand side and broken bar on right-hand side. For the first case (upper left) the rotor bar passing frequency (RBPF) and its harmonics prevail in the high frequency area, rapidly increasing their frequency as the rotor accelerates. Several family groups of harmonics can be appreciated for $k_{l}=3$ (c1), $k_{l}=2(\mathrm{c} 2)$ and $k_{1}=1(\mathrm{c} 3)$ in (7). The high frequencies they rapidly reach cause aliasing, seen as descending components in the high frequencies after 2,000 ms. Below them, the diagram is dominated by the constant twice line frequency component and its harmonics (c4) at $100 \mathrm{~Hz}, 200 \mathrm{~Hz}$ and $300 \mathrm{~Hz}$, whose amplitudes reflect electrical faults in the stator, not simulated in this work. The amplitude of the first of them is fairly constant during the entire transient.

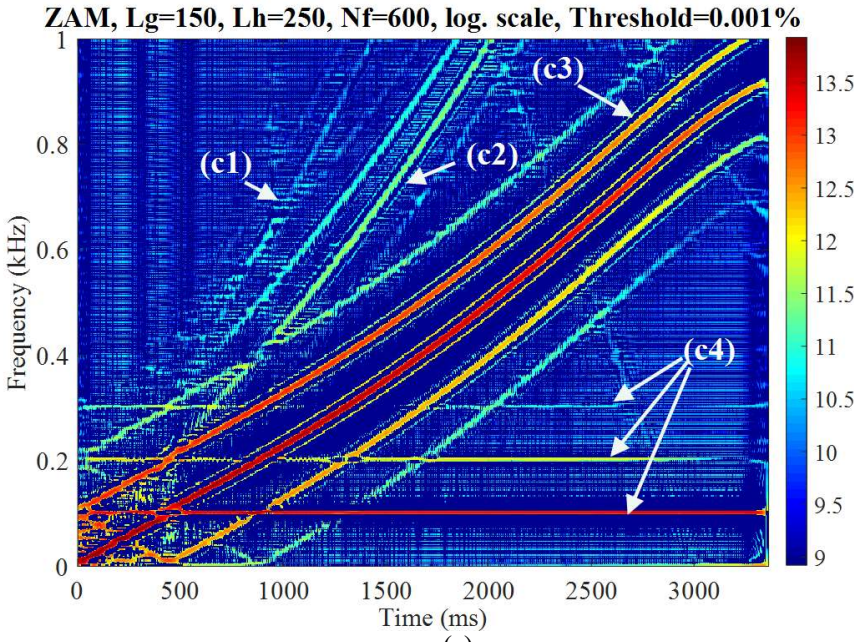

(a)

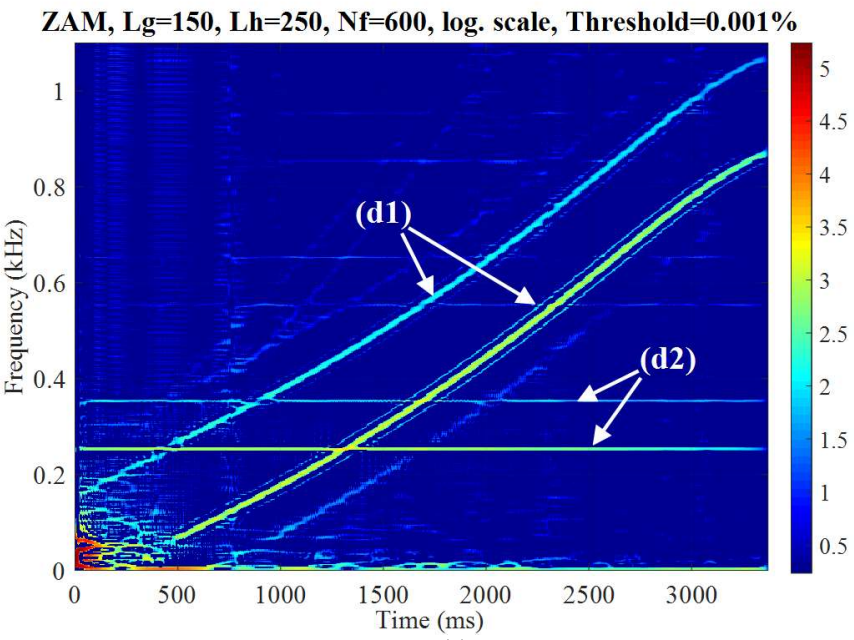

(c)

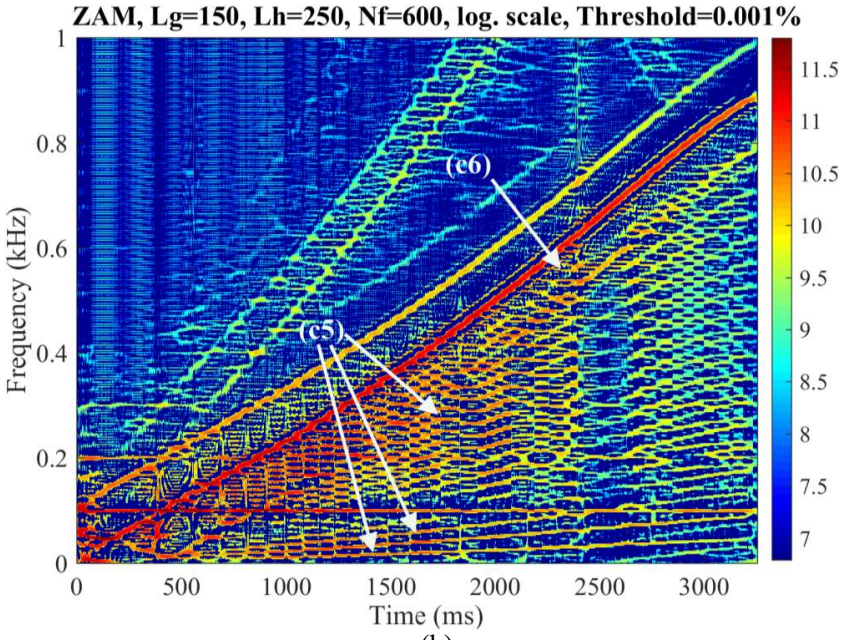

(b)

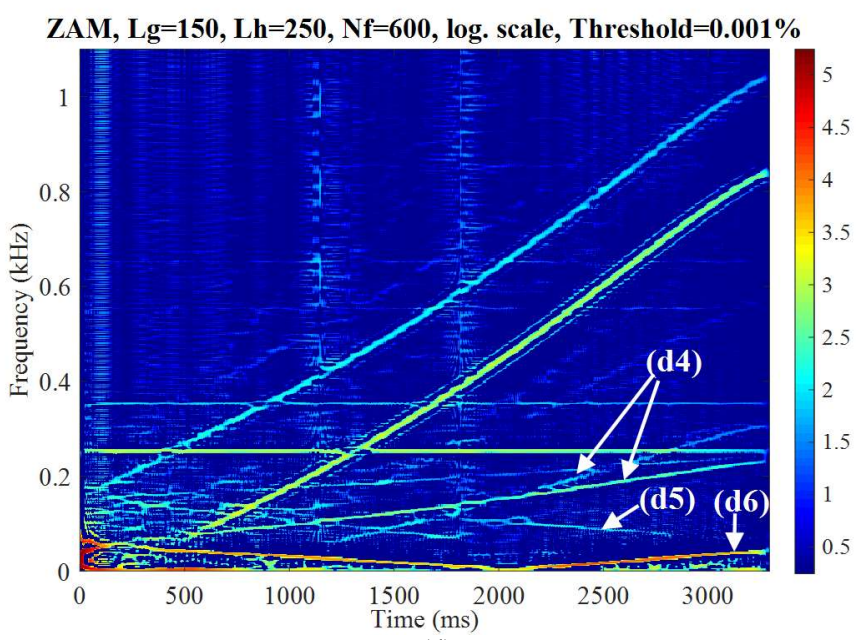

(d)

Fig. 6. ZAM distribution of the simulated stress waveform (up) for healthy (a) and broken bar (b) states, and ZAM distribution of the simulated current waveform (bottom) for healthy (c) and broken bar states (d). The rotor-speed dependent harmonics that the asymmetry adds are clearly shown. 
The upper right diagram in Fig. 6 shows the time-frequency decomposition of the simulated vibration waveform for the case of broken bar. In addition to the components depicted in the healthy state (left), rotor induced ones appear related to its rotational speed $(1 x)$ in the area below the rotor bar passing frequency (c5), according to (8) and even as one of its sidebands (c6) $\left(k_{1}=1, k_{2}=2\right.$ and $k_{3}=-1$ in (7)) as experimentally studied in [16]. In the first case it must be remarked that in an actual machine many different causes (such as eccentricity, rotor bow, etc.) can contribute to this rotating frequency harmonics; however, in a simulated machine the influence of just one fault can be evaluated. This main rotational component and its harmonics can be seen with stationary operation frequencies between $50 \mathrm{~Hz}$ and $800 \mathrm{~Hz}$. The highest amplitude corresponds to the twice line frequency vibration component, as well as to the rotor bar passing frequency, followed by the rotational frequency one $(1 x)$ and two of its harnonics $(2 x, 3 x)$.

The lower part of Fig. 6 shows the time-frequency decomposition of the currents for a healthy (left) and faulty (right-hand side) machine. With the purpose of portraying the evolution of fainter harmonics, the main current component has been filtered as in [21]. For both cases two PSH dominate the diagram $\left(k_{7}=1, k_{8}=+3,-1\right)$ as the increasing speed of the rotor leads them toward higher frequencies (d1). Using the ZAM, no other constant components need to be filtered in order to prevent crossterms, so the winding harmonics at $250 \mathrm{~Hz}$ and $350 \mathrm{~Hz}(\mathrm{~d} 2)$, as well as other weaker ones $11 f, 13 f, 17 f$ and $19 f$ can also be appreciated.

Nevertheless, when the current spectrum for the startup transient of the faulty machine is analyzed, clear components related to the rotor asymmetry appear at low frequencies: ascending ones for $k_{5} / p=3,5$ in (9) (d4), descending ones towards the suppressed main current component at $50 \mathrm{~Hz}$ (d5) and the LSH $50(1-2 s) f(\mathrm{~d} 6, \mathrm{~b} 4$ in Fig. 3), whose amplitude dominates in this case the diagram, moving from $50 \mathrm{~Hz}$ to $0 \mathrm{~Hz}$ and then back to $50 \mathrm{~Hz}$ as the slip changes from 1 to 0 . This is the harmonic commonly used to detect a rotor bar breakage by means of current analysis.

\section{Time-frequency comparison with experimental results}

In order to compare the frequency content of the simulated response of the faulty motor during the transient with experimental results, the displacements calculated in the vertical direction of Probe 1 are differentiated once. In turn, an integration performed on the experimental waveform shown in Fig. 5 (top) yields the same magnitude (speed) for both cases. Both decompositions are shown in Fig. 7 and Fig. 8 respectively. Regarding the simulated signal in Fig. 7, the effect of the derivative computation is shown as an increase of the amplitude of the higher frequencies, whilst the lower ones, below $200 \mathrm{~Hz}$, are damped. The other components are the same shown in Fig. 6 (b): the RBPF harmonics (e1), one of its sidebands (e2) and the rotational speed-related components (e3).

Fig. 8, corresponding to the experimental signal's timefrequency distribution, portrays a higher energy level and is dominated by two RBPF harmonics (7) with $k_{2}=2,-3$ (e1), their sidebands (e2) and the lower rotational speed-related components $(1 x, 3 x)(\mathrm{e} 3)$. A full family of the later, similar to what is seen in Fig. 7 develops for stationary operation (beyond $6500 \mathrm{~ms}$ ). Fainter components could not be depicted due to the noise added by the integration procedure.

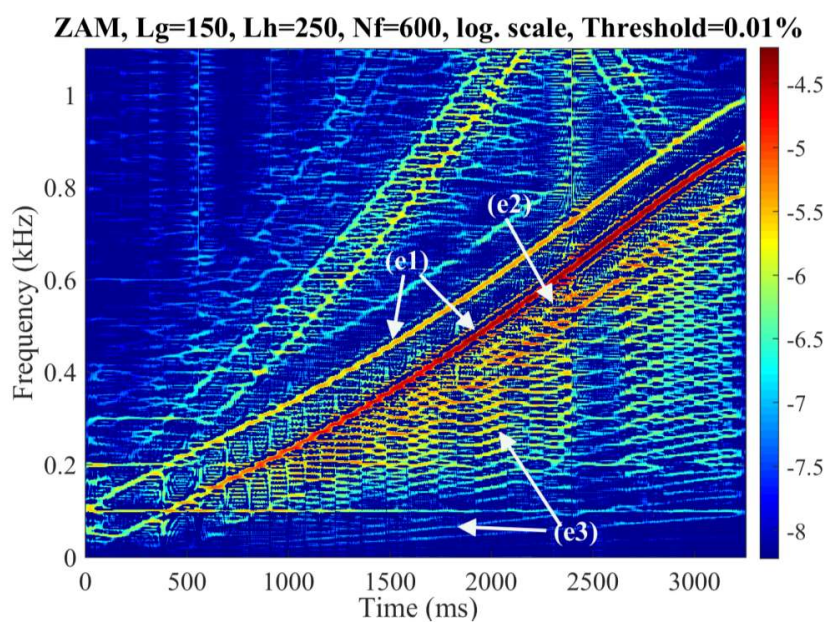

Fig. 7. ZAM distribution of the calculated speed waveform for the simulated broken bar state. The damping of lower frequencies can be appreciated compared to Fig. 6 (b).

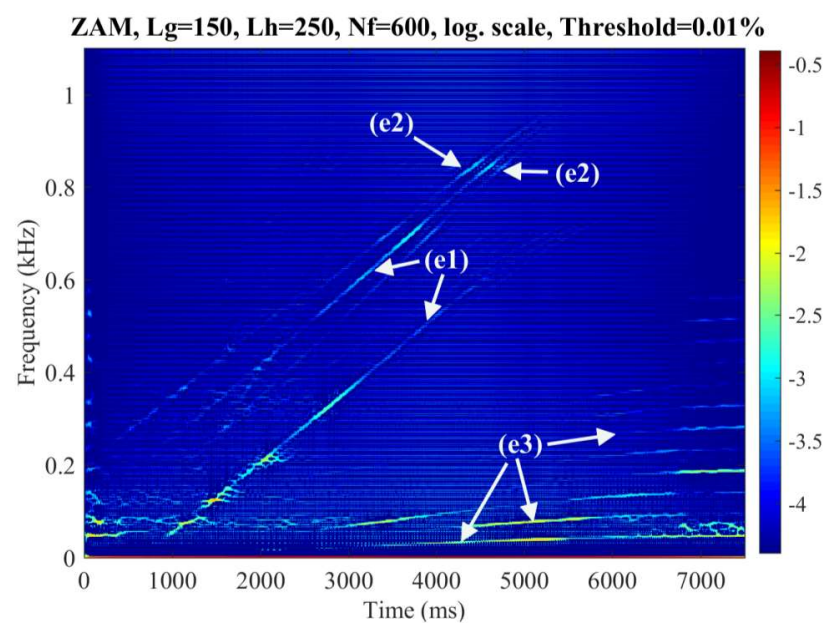

Fig. 8. ZAM distribution of the calculated speed waveform for the experimentally recorded broken bar state.

\section{Quantitative study}

Fig. 9 depicts the energy of the LSH 50 stator current component as tracked by the particle filtering method, for both simulated and experimental data of the same machine having a bar breakage [16]. The experimental test lasted longer, around 6 seconds instead of the 3.3 seconds simulated in this work, however, this shouldn't affect the values of the fault indicator. Two lobes can be appreciated in both results, corresponding to the descending and ascending branches of its evolution (Fig. 6 (d6)). Nevertheless, the energy found in the simulated case is much higher, due to the increase of the resistance for the entire bar, a limitation of 2D simulations. In the real case, interbar currents exist and contribute to damp the effect of the fault [22]. The indicator (14) yields $42.5 \mathrm{~dB}$ for the experimental case and $37 \mathrm{~dB}$ for the simulated one. 


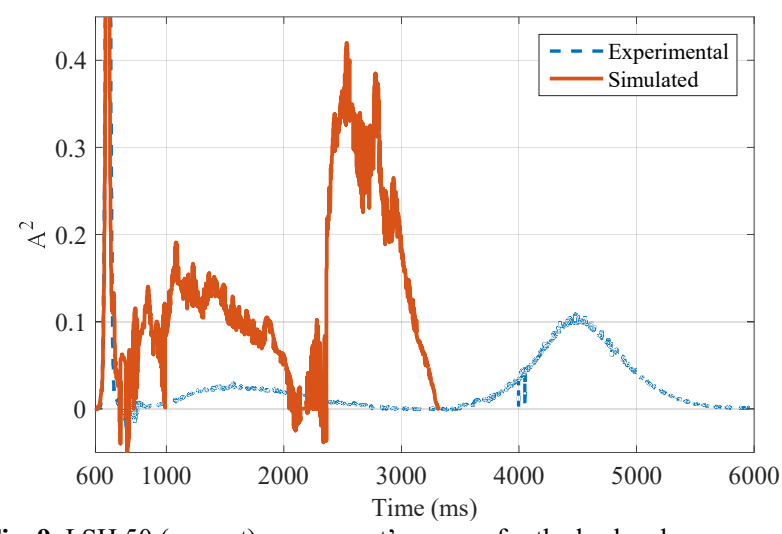

Fig. 9. LSH 50 (current) component's energy for the broken bar case.

A similar quantification procedure can be applied to the vibration component $1 x$ (Fig. 6 (c5)) evolving from $0 \mathrm{~Hz}$ to nearly $50 \mathrm{~Hz}$ during the transient and corresponding to the turning speed of the rotor. Harmonic tracking is more difficult under these conditions, in the experimental signal because of the higher noise content, and in the simulated one due to the fact that several components share the same time-frequency box. Both waveforms are thus compared for the broken bar state using acceleration, in which a good tracking of the variable frequency component was obtained in both experimental and simulated cases for the evolution of this harmonic between 20 and $45 \mathrm{~Hz}$. The fault indicator (14) yields a value of $35.8 \mathrm{~dB}$ for the experimental waveform and $78.7 \mathrm{~dB}$ when studying the simulated one, which well portrays the small contribution of the electric fault to this component, mainly affected by mechanical sources. Furthermore, Fig. 10 shows the energy profile during part of the transient for both analysis, differing greatly since the real motor crosses a resonant region before achieving stationary speed and these effects for which also the load and the support structure contribute, cannot be taken into account in a $2 \mathrm{D}$ simulation.
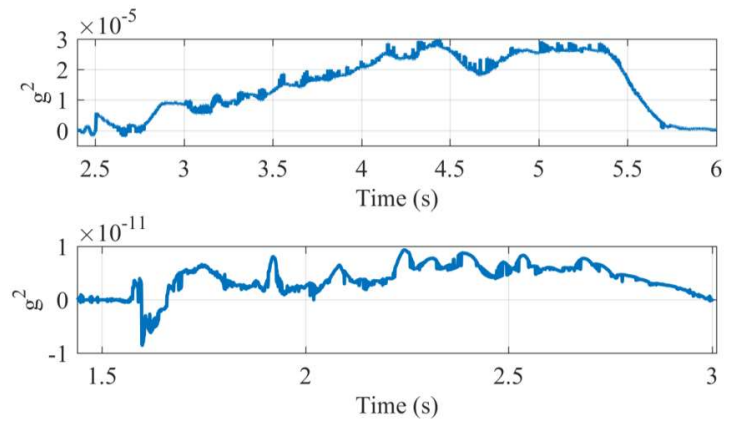

Fig. 10. Energy in the $1 x$ (vibration) component for the broken bar state. Experimental (top) and simulated (bottom).

\section{E. Discussion of the vibration results}

As shown comparing the waveforms in Fig. 4 (top and middle) and Fig. 5 (top), the proposed straightforward approach yields for the case studied an amplitude of the main components comprising the vibrational response of the stator within the same order of magnitude of the measured ones, which in some cases may be enough for ascertain the impact on this quantity of electrical faults. Furthermore, a detailed experimental study of the rotating speed-related components during the startup transient can be enhanced by employing speed sensors instead of accelerometers that tend to amplify the effect of the RBPF harmonics. The frequency composition and evolution shown in Fig. 6 (b) and Fig. 7 well matches the experimental one presented in Fig. 8, with the RBPF harmonics dominating the diagram and even depicting one of its sidebands. Nevertheless, and in spite of the complete breakage simulated, with no interbar currents that dampen the effect of the fault (Fig. 9), the rotational frequency component $(1 x)$ that is affected by this defect exhibits a negligible energy level as shown in Fig. 10, since the ground value for the time-frequency analysis of experimental signals lies above $60 \mathrm{~dB}$, and therefore, it couldn't be used to diagnose the fault studying the transient. For a DOL startup, new components that arise due to the fault, as the sidebands around the RBPF harmonics studied in [16] seem a more reliable indicator of the defect.

\section{CONCLUSIONS}

This work assesses a procedure to obtain the simulated vibration spectrum of an induction motor during transients. A strongly coupled 2D electromagnetic (for the full section of the motor) and 2D linear elastic (for the stator) procedure is able to yield amplitudes of the main components within one order of magnitude of the experimental ones and an accurate frequency evolution of several of them, including faint sidebands of high frequency harmonics, during a direct-on-line startup. The application of the Maxwell stress tensor to the inner surfaces of the stator initially suffices for evaluating the influence of electrical faults independent from other mechanical sources of vibration. However, 3D effects and resonances of the structure triggered as the rotor accelerates cannot be modelled in this way and hence the amplitudes of all these components are not accurately described.

\section{REFERENCES}

[1] A. Bellini, F. Filippetti, C. Tassoni, G.-A. Capolino, "Advances in diagnostic techniques for induction machines," IEEE Trans. Ind. Electron., Vol. 55, no. 12, pp. 4109-4126, Dec., 2008.

[2] R. Singleton, E. Strangas, S. Aviyente, "The Use of Bearing Currents and Vibrations in Lifetime Estimation of Bearings," IEEE Trans. Ind. Informat., vol. 13, no. 3, pp. 1301-1309, Jun. 2016.

[3] S. Al-Ali, R. Dabbousi, "Rotor hot spot detection and resolution in large oil and gas industry motors," in Proc. Industry Applications Society 60th Annual Petroleum and Chemical Industry Conference, Sept. 23-25. 2013, Chicago, IL, USA, pp. $1-8$.

[4] E. Hashish, K. Miller, W. Finley, S. Kreitzer, "Vibration diagnostic challenges in electric motor applications," in Proc. 2015 IEEE Petroleum and Chemical Industry Committee Conference (PCIC), 5-7 Oct. 2015, Houston, TX, USA.

[5] J. R. Cameron, W. T. Thomson, A. B. Dow, "Vibration and current monitoring for detecting airgap eccentricity in large induction motors", Proc. Inst. Elect. Eng._Elect. Power Appl., vol. 133, no. 3, pp. 155-163, May 1986.

[6] P. J. Rodriguez, A. Belahcen, and A. Arkkio, "Signatures of electrical faults in the force distribution and vibration pattern of induction motors," Proc. Inst. Elect. Eng.-Elect. Power Appl., vol. 53, no. 4, pp. 523-529, Jul. 2006.

[7] J. Martinez, A. Belahcen, J.G. Detoni, "A 2D magnetic and 3D mechanical coupled finite element model for the study of the dynamic vibrations in the stator of induction motors," Mechanical Systems and Signal Processing, vol. 66-67, pp. 640-656, Jan. 2016. 
[8] J. Martinez, A. Belahcen, A. Muetze, "Analysis of the vibration magnitude of an induction motor with different numbers of broken bars," IEEE Trans. Ind. Appl., vol. 53, no. 3, pp. 2711-2720, May-Jun. 2017.

[9] S. G. Ghalamestani; L. Vandevelde; J. A. A. Melkebeek, "Magnetic forces and magnetostriction in rotating electrical machines," in Proc. XXII International Conference on Electrical Machines (ICEM), Sept. 4-7, 2016, Lausanne, Switzerland.

[10] S. Elder, J. F. Watson, and W. T. Thomson, "Fault detection in induction motors as a result of transient analysis," in Proc. 4th Int. Conf. Elect. Mach. Drives, London, U.K., 1989, pp. 182-186.

[11] S. H. Kia, H. Henao, and G. A. Capolino, "Diagnosis of broken-bar fault in induction machines using discrete wavelet transform without slip estimation," IEEE Trans. Ind. Appl., vol. 45, no. 4, pp. 1395-1404, Jul./Aug. 2009.

[12] M. J. Picazo-Ródenas, J. Antonino-Daviu, V. Climente-Alarcon, R. Royo-Pastor; A. Mota-Villar, Combination of Noninvasive Approaches for General Assessment of Induction Motors, IEEE Trans. Ind. Appl., vol. 51, no. 3, pp. 2172 - 2180, May-Jun. 2015.

[13] F. Briz, M. W. Degnert, P. Garcia, and D. Bragado, "Broken rotor bar detection in line-fed induction machines using complex wavelet analysis of startup transients," IEEE Trans. Ind. Appl., vol. 44, no. 3, pp. 760-768, May/Jun. 2008.

[14] M. Blodt, D. Bonacci, J. Regnier, M. Chabert, J. Faucher, "On-line monitoring of mechanical faults in variable-speed induction motor drives using the Wigner distribution," IEEE Trans. Ind. Electron., vol. 55, no. 2, pp. 522-533, Feb. 2008.

[15] S. Rajagopalan, J. M. Aller, J. A. Restrepo, T. G. Habetler, R. G. Harley, "Detection of rotor faults in brushless DC motors operating under nonstationary conditions," IEEE Trans. Ind. Appl., vol. 42, no. 6, pp. 1464-1477, Nov./Dec. 2006.
[16] V. Climente-Alarcon, J. Antonino-Daviu, F. Vedreno-Santos, R. PuchePanadero, "Vibration transient detection of broken bars by PSH sidebands," IEEE Trans. Ind. Appl., Vol. 49, No. 6, pp. 2576-2582, Nov/Dic 2013.

[17] Y. Gritli, A. O. Di Tommaso, R. Miceli, F. Filippetti, C. Rossi, Vibration signature analysis for rotor broken bar diagnosis in double cage induction motor drives, in Proc. 4th International Conference on Power Engineering, Energy and Electrical Drives, Riga, Latvia, May 13-17, 2013, pp. $1814-1820$.

[18] V. Climente-Alarcon, A. Arkkio, J. A. Antonino-Daviu, "2-D Magnetomechanical Transient Simulation of a Motor with a Bar Breakage", in Proc. 11th IEEE International Symposium on Diagnostics, Electric Machines, Power Electronics and Drives (SDEMPED), Tinos, Greece, Aug. 29-Sep. 1, 2017.

[19] R. Burnett, J. F. Watson, S. Elder, "The application of modern signal processing techniques for use in rotor fault detection and location within three-phase induction motors," Signal Processing, vol. 49, No. 1, pp. 5770, Feb. 1996.

[20] A. Bellini, F. Filippetti, G. Franceschini, C. Tassoni, G.B. Kliman, "Quantitative Evaluation of Induction Motor Broken Bars by Means of Electrical Signature Analysis“, IEEE Trans. Ind. Appl., Vol. 37, no. 5, pp 1248-1255, Sep./Oct. 2001.

[21] V. Climente-Alarcon; J. A. Antonino-Daviu; A. Haavisto; A. Arkkio, "Diagnosis of Induction Motors Under Varying Speed Operation by Principal Slot Harmonic Tracking," IEEE Trans. Ind. Appl., vol. 51, no. 5, pp. 3591 - 3599, Sep./Oct. 2015.

[22] I. Kerszenbaum; C. F. Landy, “The Existence of Large Inter-Bar Currents in Three Phase Squirrel Cage Motors with Rotor-Bar And/Or End-Ring Faults," IEEE Transactions on Power Apparatus and Systems, Vol. PAS103 , no. 7 , pp. $1854-1862$, July 1984 